\title{
Féeries
}

Études sur le conte merveilleux, XVII $-\mathrm{XIX}{ }^{\mathrm{e}}$ siècle

\section{Le conte schwobien ou la poétique du singulier}

Schwob's Tales, the Poetics of Singularity

\section{Pauline Bloch et Michel Viegnes}

\section{(2) OpenEdition}

Journals

Édition électronique

URL : http://journals.openedition.org/feeries/1051

ISSN : 1957-7753

Éditeur

UGA Éditions/Université Grenoble Alpes

Édition imprimée

ISBN : 978-2-37747-012-9

ISSN : 1766-2842

\section{Référence électronique}

Pauline Bloch et Michel Viegnes, «Le conte schwobien ou la poétique du singulier », Féeries [En ligne],

14 | 2017, mis en ligne le 31 juillet 2017, consulté le 08 septembre 2020. URL : http://

journals.openedition.org/feeries/1051

Ce document a été généré automatiquement le 8 septembre 2020.

(c) Féeries 


\section{Le conte schwobien ou la poétique du singulier}

Schwob's Tales, the Poetics of Singularity

Pauline Bloch et Michel Viegnes

"One's-self I sing, a simple separate person, Yet utter the word Democratic, the word En-Masse. of physiology from top to toe I sing, Not physiognomy alone nor brain alone is worthy for

the Muse. "

Walt WHITMAN,

" One's-self I sing », The Grass of Leaves, 1891-1892.

«Connaître le monde, c'est savoir qu'il n'y a point

d'identités formelles. "

Remy DE GOURMONT,

« Marcel Schwob », Le deuxième livre des masques,

1898.

Qu'elle se décline sous forme de préfaces ou d'essais, la réflexion poétique de Marcel Schwob se revendique invariablement d'un art qui puise son inspiration dans la multiplicité infinie du matériau vivant. Pour singulière qu'elle soit, la place de Schwob n'en demeure pas moins significative dans le champ artistique d'une époque qui pressent que le basculement dans le $\mathrm{xx}^{\mathrm{e}}$ siècle ne se fera pas sans une révolution dans la théorie du langage. La fin de siècle, lourdement héritière d'une pensée matérialiste qui a trouvé sa caution littéraire dans la production naturaliste, est à ce titre un véritable laboratoire critique auquel la réflexion schwobienne a largement contribué, au sujet du langage et de sa fonction représentative ; vivement rétif à une conception de l'art qui véhiculerait des schémas scientistes et se mettrait au service de la logique, l'écrivain tente de redéfinir une littérature qu'il entrevoit comme mimèsis du monde vivant: redéfinition d'un réalisme qui embrasse, sans le rendre exclusif, le caractère contingent du réel et la part singulière de tout être. Schwob parvient à dépasser l'antinomie entre la raison suffisante des mots et l'« universel reportage » décrié par le maître de la rue 
de Rome, en appréhendant le monde comme un vaste jeu de «signes et signes de signes $^{1}$ ", où d'autres dichotomies supposées, comme celle entre fiction et réalité historique, sont également neutralisées.

\section{De la conception schwobienne de la poéticité}

2 En effet, l'œuvre de Marcel Schwob, au regard de sa forme comme de son contenu, est une exploration d'un réel dont la structure sémiotique, complexe et dédoublée, invite non pas à la seule recherche du sens, mais à l'extension des manières d'appréhender le monde et de l'interpréter. La réalité peut prendre plusieurs visages - ou masques dans la mesure où chaque histoire a droit à son propre " langage ». Le lecteur l'éprouve dans la multiplicité des procédés littéraires qu'il adopte dans ses fictions, dans la modulation des catégories générales auxquelles appartient chacun des textes, dans la diversité des intertextes qu'il convoque, ou encore dans les conceptions philosophicoesthétiques qui caractérisent sa production théorique. L'œuvre dans son ensemble se fait l'écho d'une réflexion duelle, construite sur un rapport de forces qui confronte pour mieux les confondre la logique au paradoxe.

Considérant qu'il n'y a pas de réel en dehors de l'appréhension qu'on en a, l'écrivain conçoit l'univers comme un support fait de signes qui se donnent à voir tout autant qu'à lire, rendant compte, comme Alexandre Gefen l'exprime avec justesse, d'une " vision textualiste du monde ${ }^{2}$ ». Le "Soi-même et en masse ${ }^{3}$ ", devise de Walt Whitman à laquelle Schwob souscrit dans la préface de Coeur double - texte hautement important, puisque l'auteur y jette les bases de son esthétique - représente pour lui le modèle d'une littérature qui affirme et embrasse le caractère duel de la nature et de l'homme, qui en tant qu'objets de pensée sont toujours à considérer sous un double rapport, celui de l'un et du multiple, de l'individu et de la société, du nécessaire et du contingent.

Dans cette même préface, il propose de tracer une voie nouvelle, celle d'une écriture vouée à faire « la synthèse [...] d'un être vivant ${ }^{4}$ » - «Par vivants, entendez individuels ${ }^{5}$ » précise encore l'écrivain dans la préface des Vies imaginaires, prolongeant ainsi la réflexion du singulier entamée dans Coeur double. C'est cette conception à la fois poétique et éthique de la mimèsis que nous désignons par singularité chez Schwob: l'«objet de la représentation artistique ${ }^{6}$ " est celui de la vie, dans sa dimension contingente et ponctuelle (au sens d'une existence individuelle appréhendée à un point précis de l'espace et du temps), comprise dans la chaîne de toutes les autres. Cet individu dont l'œuvre schwobienne fait le portrait est une "singularité quelconque ", au sens où l'entend Giorgio Agamben, c'est-à-dire une singularité «pr[ise] seulement dans son être telle qu'elle est ${ }^{7}$ ", saisie par les sens et dégagée de toute raison et de toute justification". À l'évidence, Schwob s'inscrit pleinement dans une démarche antinaturaliste et antiscientiste qui réunit sous la même bannière la génération des écrivains fin-de-siècle. C'est à ce titre notamment que l'on peut examiner la préface de Couur double en sa qualité de manifeste littéraire: elle propose en effet une nouvelle forme d'écriture fictionnelle - le "roman d'aventures " (qui transcende sa pure définition générique, puisque Schwob n'a à proprement parler jamais écrit de roman) - débarrassée du fardeau documentaliste et déterministe qui avait poussé Zola et les siens, aux dires de Huysmans, à "n'admett[re] guère [...] l'exception » et à se confiner « dans la peinture de l'existence commune [...] sous prétexte de faire vivant ${ }^{10}$ ». Chez Schwob, l'être (re)construit par le langage, libéré du regard déterministe et 
généraliste de l'analyse scientifique, n'est plus soumis aux lois de la causalité, ni à l'orientation d'un sens unique :

Les êtres vivants, spontanés, libres, dont la synthèse psychologique et physiologique, malgré certaines conditions déterminées, dépendra des séries qu'ils rencontreront, des milieux qu'ils traverseront, tels seront les objets de l'art. Ils ont des facultés de nutrition, d'absorption et d'assimilation; mais il faut tenir compte du jeu compliqué des lois naturelles et sociales, que nous appelons hasard, que l'artiste n'a pas à analyser, qui est véritablement pour lui le Hasard, et qui amène à l'organisme physique et conscient les choses dont il peut se nourrir, qu'il peut absorber et s'assimiler ${ }^{11}$.

5 Car si c'est à la synthèse d'un être vivant, et donc de l'individu, que la littérature schwobienne aspire, c'est bien d'abord l'exercice logique de l'analyse qui tombe sous la critique du jeune préfacier de Couur double, en ce qu'elle empêche, par une pratique systématique de la décomposition et de l'«énumération ${ }^{12}$ ", la spontanéité de l'impression qui participe au plaisir esthétique du lecteur :

L'analyse des passions dans la description des héros ou dans le rôle des acteurs est déjà une pénétration de l'art par la critique. L'examen que la personne représentée fait d'elle-même provoque un examen imité chez le spectateur. Il perd la sincérité de ses impressions ; il ratiocine, discute, compare $[. . .]^{13}$.

Dans un essai écrit deux ans plus tôt et justement intitulé «Le réalisme », Schwob plaide ainsi pour un "vrai " réalisme (qui n'est pas à confondre avec l'école proprement dite) qui, libéré du joug déterministe, deviendra un « impressionnisme » :

Il y a entre ces deux formules [le réalisme objectif et le réalisme subjectif] place pour le vrai réalisme - celui qui n'a pas prétentions scientifiques, qui ne recherche pas le lien des causes efficientes. Ce sera l'impressionnisme ; il s'agira d'imiter la nature dans les formes que nous saisissons en elle ${ }^{14}$.

7 L'art n'est pas la nature; il est une représentation et, dans la mesure où il suppose de la « reproduire artificiellement ${ }^{15}$ », il est contraint d'adopter " une composition spéciale » - que l'écrivain baptise «symétrie»- subsumant toute idée de genre. Synonyme d'équilibre et d'harmonie («l'âme [doit] être en quelque manière une harmonie, une chose symétrique et équilibrée $\left.{ }^{16} »\right)$, la symétrie est le principe qui sous-tend l'élaboration d'une esthétique impressionniste: elle vise à donner une impression de l'homme dans sa dimension dynamique. Comme la peinture, l'écriture tourne le regard vers les multiples fluctuations de l'instant. Nées de l'observation des «crises» de l'homme, de ces moments où la vie est sur le point de basculer, les impressions qui submergent le lecteur font de l'art une expérience fondamentalement sensible et plurielle, susceptible «de faire pressentir que dans une seconde de vie intense nous revivons virtuellement et actuellement l'univers ${ }^{17}$ ».

8 L'artiste n'est donc pas soumis à l'autorité du fait ; il est libre de choisir le moment et la manière de traiter le phénomène. Aussi ne semble-t-il pas contradictoire que Schwob affirme la liberté absolue d'un art de «l'Idée [...] fixe et immobile ${ }^{18} »$. L'emploi de la capitale est ici important: $i \delta \varepsilon \alpha^{19}$, la racine grecque du mot, se définit par «forme visible, aspect ». On sait, grâce aux nombreuses études qu'il consacre à Stevenson - et nous allons nous y intéresser plus loin - l'importance que Schwob accorde à l'image et il ne paraît pas anodin que le recueil de Cour double soit précisément dédié à son modèle écossais. Ce n'est donc pas de la rigidité d'une conception de l'art qu'il est ici question, mais de la fixité inhérente à l'image - «l'Idée » :

Il suffit de lire Eschyle avec quelque attention pour percevoir cette permanente symétrie qui est le principe de son art. La fin des pièces est pour lui une rupture de 
l'équilibre dramatique. La tragédie est une crise, et sa solution une accalmie. En même temps, à Égine, un peu plus tard à Olympie, des sculpteurs de génie, obéissant aux mêmes principes d'art, ornaient les frontons des temples de figures humaines et de compositions scéniques symétriquement groupées des deux côtés d'une rupture d'harmonie centrale. Les crises des attitudes, réelles mais immobiles, sont placées dans une composition dont le total explique chacune des parties ${ }^{20}$.

L'art symétrique se donne pour mission de capter ce qui se dérobe d'ordinaire au regard, ces multiples singularités de l'instant, et de les donner à voir quand l'art réaliste cherche à décrire de manière objective les phénomènes dans leur enchaînement causal. Schwob exprime d'ailleurs cette difficulté quasi insurmontable à saisir par les seuls outils de l'intellect la nature des sensations qui se jouent dans l'homme :

Il m'est impossible de m'entendre avec les psychologues [...]. La vie ne me parait pas un thème de compositions de classe. Partout où je cherche à la saisir, elle fait explosion sous mes mains. J'y vois une succession de crises causées par un mouvement ascendant vers l'organisation et l'individualisation d'une part, et par un mouvement en sens contraire vers la dégénération et la désintégration de l'autre. Et c'est un ensemble de phénomènes portés à leur tension la plus haute, puis ramenés à un état d'indifférence complète ${ }^{21}$.

10 Les diverses émotions, comprises tout entières dans un récit resserré, permettent «l'équilibre dramatique » et contribuent ainsi à l'harmonie esthétique. Christian Berg rappelle à ce titre que Schwob comprend la notion de symétrie en fonction de sa définition originelle :

Dans une dissertation sur "Eschyle et Aristophane ", qui date de 1889 ou de 1890, Schwob définit la symétrie comme la «recherche exclusive de l'harmonie des rapports dans la forme ». Dans son approche, le jeune universitaire reste fidèle au sens étymologique du mot qui doit son origine au grec metron (mesure) enrichi du préfixe sym qui indique qu'il s'agit du rapport de mesure entre plusieurs - au moins deux - formes ou objets. Le mot répond donc aux idées de mesure, de proportion, d'harmonie, de rapports heureux entre les parties et le tout [... $]^{22}$.

11 L'image la plus saisissante, source d'harmonie et d'équilibre parce que synthétisant des impressions complexes et souvent contraires, devient ainsi selon Schwob une «image irréelle ", «quintessence de la réalité ${ }^{23}$ ", et c'est la raison pour laquelle la frontière entre réalité et imaginaire, mais surtout entre poésie et fiction, tend à s'estomper :

[...] je voudrais faire remarquer ici [...] que c'est une vérité universelle pour toutes les œuvres dignes de ce nom qu'elles ont été suggérées par la nature, par la vie, par la réalité. Et il n'y a pas de grande création poétique, si dégagée puisse-t-elle paraitre des contingences, pour peu qu'elle exerce sur nous un pouvoir d'attrait ou d'émotion, dont la force n'ait été puisée dans l'éternelle source d'énergie qu'est le monde extérieur.

Les œuvres dites «d'imagination » peu à peu viennent se classer parmi les œuvres dites « réalistes ». En ce sens, il n'y a pas de vraie distinction entre elles ${ }^{24}$.

12 Aussi Schwob ne conçoit-il pas l'art comme une représentation objective du réel qui satisferait à la seule logique. Le plaisir esthétique naît fondamentalement de l'émotion que suscite l'œuvre : en invoquant les Anciens, il observe qu'« il n'y avait pas [chez eux] de moralité dans l'art; il y avait à faire l'équilibre dans l'âme ${ }^{25}$ ». Peindre les crises de l'homme, reconnaître les tensions inhérentes à la contingence du réel, voilà l'objet propre à une expression poétique qui, transcendant l'idée générique du concept de poésie, correspond non seulement à ce que Schwob observe dans les tragédies d'Eschyle, les versets de Whitman ou les romans de Stevenson, mais aussi à ce qui sous- 
tend les contes poétiques en prose que réunissent ses deux premiers recueils. Vers et prose ne sont dès lors que des manières diverses d'exprimer l'harmonie d'un univers fondé, comme la musique, sur un système de polarités et sur des alternances de tension et de détente. Aux littérateurs britanniques qui n'accordent pas qu'on puisse traduire ${ }^{26}$ Shakespeare, dès lors que « la grâce de sa poésie disparaît, disent-ils, parmi la prose » et " qu'un vers français ne saurait représenter un vers anglais », Schwob rétorque :

C'est vrai ; mais le graveur qui fait une eau-forte d'après un tableau n'y transpose pas les couleurs. Il les transpose en valeurs. Si on peut comparer la peinture et la poésie, il faut accorder qu'un poème mis en prose est comme un tableau mis en gravure. Le poème perd le mystère de son harmonie et le tableau la brume de ses teintes; en échange la prose donne la gloire du verbe et l'eau-forte le tranchant éclat de ses lignes. Tout art est une interprétation; et si la nature peut être interprétée, l'œuvre du poète ou du peintre sont-elles plus rebelles ${ }^{27}$ ?

13 La poésie n'est pas l'apanage du Voyant capable de lever le voile de l'univers et d'en déchiffrer le sens. Comme il le dit dans un article sur Verlaine, «ils [les poètes] ne crochètent pas les serrures des choses pour en faire jaillir des symboles. Car les choses n'ont pas toujours à être des symboles. Ce sont souvent de pauvres choses muettes, auxquelles on ne doit pas faire parler un langage faux ${ }^{28} »$. L'artiste est un interprète actif des signes du réel dans la mesure où il les lit et les transcrit au moyen de son imagination ${ }^{29}$. Cette conception, qui témoigne du caractère sémiotique du réel, admet - Schwob en pressent-il déjà la description saussurienne ? - la double dimension du signe, sensible et conceptuelle. Faisant montre de la part arbitraire du vivant et de ses contradictions inhérentes, la poésie s'opposerait ainsi à une appréhension purement déterministe de l'humain: crises, tensions et paradoxes, voilà ce que figure le «processus de contraste symétrique » et sur quoi repose la « loi esthétique ${ }^{30}$ » (le terme même est vecteur de tension) qui régit le langage poétique. Jean-Pierre Bertrand remarque très justement que les récits de coeur double ont pour point commun un matériau fictionnel construit sur un régime de sens qui, du point de vue de la logique, relève de l'aporie :

Dans chacun de ses contes, Schwob remet ainsi en cause toute pensée duale et dichotomique : le soi contient toujours l'autre, l'unique contient toujours le double et vice versa. Les sciences du langage, auxquelles Schwob, en bon disciple de Bréal et de Saussure [...], fut si attentif, et qui, d'une certaine façon, se substituèrent dans l'imaginaire littéraire au modèle physiologique, donnaient raison à cette nouvelle dialectique qui incluait au lieu d'exclure et scindait sans diviser ${ }^{31}$.

Le texte schwobien - comme le texte poétique en général - n'échappe d'ailleurs pas, nous venons de le voir, aux lois de la «composition » qui permettent d'en assurer la cohérence. Au sujet du poème en prose, qu'il est parfois difficile de distinguer du conte poétique en prose, Yves Vadé interroge le principe organisateur, condition essentielle de tout texte poétique :

Les poèmes en prose ne se sont pas contentés [...] de soustraire leurs textes au principe de l'équivalence analogique et homogénéisante qui régit l'écriture en vers. Ils ont, d'une manière toute empirique et sans appui d'aucune théorie, substitué à ce principe un principe contraire, de nature non plus formelle mais sémantique, et que l'on pourrait nommer un principe de tension : un poème en prose n'est pas une page de prose ordinaire parce que cette page est tendue entre deux pôles contraires, dont l'opposition commande toute l'organisation du texte ${ }^{32}$.

Si l'impressionnisme de Schwob apparaît comme une littérature du dévoiement, c'est parce que, n'ayant de cesse d'interroger - esthétiquement et pragmatiquement - les limites du sens et démultipliant les usages de la fonction herméneutique du langage, il 
accorde, avec une optique d'une rare modernité, une responsabilité majeure à l'acte de lecture. Le conte tel que le pratique Schwob, en position instable entre mimèsis et absence de représentation, prend ainsi une teinte poétique dès lors qu'il "tire", comme l'observe Bertrand Vibert, « son mystère, sa réserve de sens ou sa signifiance de cette représentation même ${ }^{33}$ ».

\section{Le sens : construction nécessaire du sens et valeur relative}

16 Dans la fameuse préface du Roi au masque d'or, Schwob, par une volonté qui semble dépasser le seul désir d'« excuser la composition de [son] livre ${ }^{34}$ ", s'exprime au sujet de la valeur relative que présente notre appréhension singulière du réel et du signe, en évoquant le caractère perspectiviste de la différence et de la ressemblance ( «la ressemblance et la différence sont des points de vue »), mais également au sujet de la dimension inintelligible du caractère sériel et fragmentaire du réel. Celui-ci ne nous apparaît que tel que l'on est disposé à le considérer et chaque objet qui le compose ne s'observe et ne se comprend que dans la mesure où il entretient un rapport de continuité ou de discontinuité avec les autres objets. Nous rencontrons ainsi le problème de la singularité, celui-ci, comme le souligne clément Rosset, étant «bien connu de l'histoire de la philosophie, qui a toujours achoppé sur le caractère impensable et indescriptible de la notion de même dès lors qu'il n'est aucun autre pour en rendre raison ${ }^{35}$ ». Mais ceci fait également écho à la question évoquée dans la préface de Cour double, celle du double aspect du monde, contingent sous le regard discriminant de l'imaginaire et déterminé sous le regard généralisant de la raison. Le réel est un langage sujet à interprétation: ressemblance et différence sont bien équivalents puisqu'ils sont les deux aspects sous lesquels chaque chose se donne à lire en tant que signe. Comme l'indique Christian Berg, Schwob rappelle «l'infini engendrement des signes par eux-mêmes » où «le signifié s'envisage du point de vue de la généralité et donc de la ressemblance », et où « le référent concret s'envisage du point de vue de la particularité et donc de la différence ${ }^{36}$ ». Les signes n'ont donc qu'une existence abstraite, renvoyant toujours à autre chose qu'eux-mêmes ${ }^{37}$. C'est là où l'interprétation, qui n'est qu'un nouveau système de signes, rencontre ses limites :

Toutes choses ont entre elles des rapports. Quand nous saisissons leurs rapports de position, nous les classons suivant la cause et l'effet. Quand nous les concevons selon leurs relations de ressemblance et de grandeur, nous les classons suivant les idées logiques de notre esprit. Ces notions étant communes à tous les philosophes, il $\mathrm{y}$ a fort à parier qu'elles ne suffisent pas à la vérité. On peut imaginer que les choses ont entre elles d'autres rapports que le rapport scientifique et le rapport logique. Elles peuvent se rapporter l'une à l'autre en tant qu'elles sont des signes, car les signes n'ont ni quantité ni qualité absolue. Et il est possible que les signes étant très différents, les choses signifiées soient très voisines. De ces choses signifiées les sens ni l'intelligence ne peuvent rien savoir ${ }^{38}$.

Conscient du défi que suscite l'ambition d'une littérature qui joint à des principes esthétiques des considérations philosophiques, l'écrivain tire parti de l'espace que lui réservent ses préfaces pour évoquer la part ineffable de l'objet qu'il se propose de traduire dans son écriture (à propos de Stevenson, Schwob dira que chez lui «l'art consiste [...] à ne point dire $\left.{ }^{39} »\right)$. Ce faisant, il s'expose au risque de chercher à traduire, avec un langage qui fonctionne essentiellement sur un modèle logique, ce qui relève 
précisément du domaine de l'indicible. Cette difficulté ne doit cependant pas nous conduire à écarter la production théorique de l'écrivain. Au contraire, elle nous apporte justement un éclairage sur la manière dont nous pouvons aborder une œuvre fictionnelle qui contraint le lecteur à redéfinir la valeur de l'interprétation. Le parcours herméneutique devrait désormais pouvoir s'accommoder, notamment dans le sillage de la pensée nietzschéenne, de son incapacité à déterminer entièrement le sens de l'objet de son étude et renoncer à toucher à une vérité qui se cacherait entre les lignes du texte. Pour Schwob en effet, il semble nécessaire de rappeler une évidence à laquelle on peine souvent à consentir, à savoir que le réel n'est jamais appréhendé autrement que par un point de vue particulier. C'est pourquoi l'écrivain porte autant d'attention à l' impression que suscite le texte chez le lecteur et en fait la condition d'un réalisme "supérieur", comme le relevait déjà Georges Trembley, l'un des premiers commentateurs de Schwob ${ }^{40}$. Pour Michael Riffaterre, «ce n'est pas dans l'auteur [...] ni dans le texte isolé que se trouve le lieu du phénomène littéraire, mais [...] dans une dialectique entre le texte et le lecteur ${ }^{41} »$.

Dans la préface qui ouvre le recueil du Roi au masque d'or, Schwob interpelle précisément le rôle de ce lecteur. En donnant la parole à un observateur "supérieur " et étranger ${ }^{42}$, l'écrivain s'accorde la liberté de partager ses convictions esthétiques et de redéfinir le dessein de la lecture, puisque son lecteur idéal est capable de combiner ce point de vue dédoublé auquel Schwob aspire tant, « la vue bornée d'un artiste en même temps que la généralisation d'un savant ${ }^{43} »$ :

Saisissez les différences charmantes par votre imagination, mais apprenez à les confondre en la continuité des ressemblances, qui font les lois explicatives, par l'exercice de votre raison. Ne donnez pas plus de foi à ceux qui vous montrent la discontinuité, ou les différences individuelles, ou la liberté dans l'univers, qu'à ceux qui vous exposent sa continuité ou ses lois nécessaires [...] Imaginez que la ressemblance est le langage intellectuel des différences, que les différences sont le langage sensible de la ressemblance. Sachez que tout en ce monde n'est que signes, et signes de signes ${ }^{44}$.

19 Au regard du signifié donc, les objets et individus du monde, dont la présence demeure énigmatique, renvoient toujours à un sens qui les dépasse. Les signes, comme les choses, ne se donnent à lire que sous le mode de l'apparaître. L'influence nietzschéenne semble toute proche ${ }^{45}$; Schwob conçoit, à l'instar du philosophe, que le point de vue est la condition de la manifestation du réel puisque notre capacité à appréhender les objets $\mathrm{du}$ monde se limite immanquablement à une lecture superficielle : "Ainsi que nousmêmes, elles [les choses] sont les masques de visages éternellement obscurs ${ }^{46}$. »

Rappelons que par cette préface, Schwob cherche à excuser la composition singulière de son recueil. Ceci n'est pas aussi opportun qu'il n'y paraît. Transposé à l'échelle de la création humaine, l'exposé philosophique auquel il convie le lecteur et par lequel il l'invite à considérer le monde sous les seules données du langage devient un plaidoyer pour la liberté artistique ; à l'image d'un démiurge, l'artiste, qui essaie d'interpréter les choses du monde "sur le modèle même que Dieu a imaginé de les proférer ", compose lui aussi un univers fait de signes appelant à se lire selon les deux perspectives qui s'offrent à l'observateur humain, celle de la raison et celle de l'imaginaire. Langage du monde, les objets sont à la fois signifiant et signifié :

Si vous pouvez supposer un Dieu qui ne soit pas votre personne et une parole qui ne soit pas la vôtre, concevez que Dieu parle : alors l'univers est son langage. Il n'est pas nécessaire qu'il nous parle. Nous ignorons à qui il s'adresse. Mais ses choses tentent de nous parler à leur tour, et nous, qui en faisons partie, nous essayons de 
les comprendre sur le modèle même que Dieu a imaginé de les proférer. Elles ne sont que des signes, et des signes de signes. Ainsi que nous-mêmes, ce sont les masques de visages éternellement obscurs. Comme les masques sont le signe qu'il y a des visages, les mots sont les signes qu'il y a des choses. Et ces choses sont les signes de l'incompréhensible. Nos sens perfectionnés nous permettent de les disjoindre et notre raisonnement les calcule sous une forme continue $[. . .]^{47}$.

Aussi la pensée schwobienne concède-t-elle bien à la lecture, à la fois comme expérience sensible et comme interprétation, une part essentielle dans l'élaboration du sens de l'œuvre. Schwob ne le dit pas autrement, lorsqu'il fait remarquer que le réel, qu'il s'agisse du monde empirique ou du monde imaginaire, se présente toujours sous la forme d'un langage à traduire : «Tout art est interprétation; et si la nature peut être interprétée, l'œuvre du poète ou du peintre sont-elles plus rebelles ${ }^{48}$ ? »

Redoublement du signe, la représentation n'échappe pas à l'interprétation. C'est pourquoi toute lecture sincère suppose selon Schwob un engagement, libre et joyeux, qui se situe d'abord au niveau de l'imagination:

Le vrai lecteur construit presque autant que l'auteur : seulement il bâtit entre les lignes. Celui qui ne sait pas lire dans le blanc des pages ne sera jamais bon gourmet de livres. La vue des mots comme le son des notes dans une symphonie amène une procession d'images qui vous conduit avec elles ${ }^{49}$.

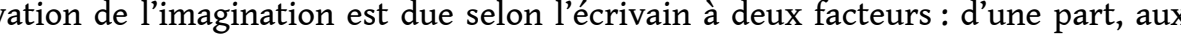
blancs du texte, que Schwob désigne comme les «silences du récit ${ }^{50}$ " appelant à la manière $\mathrm{du}$ vide l'imagination propre au lecteur et, d'autre part, aux analogies spontanées que certains mots peuvent suggérer dans son esprit ${ }^{51}$. C'est en multipliant les lieux d'indétermination du texte et en faisant, comme nous allons le voir, un usage particulier de l'image, que l'écrivain exerce son pouvoir créatif. Toute œuvre, même biographique, suppose l'intrusion de l'imaginaire :

L'art du biographe consiste justement dans le choix. Il n'a pas à se préoccuper d'être vrai ; il doit créer dans un chaos de traits humains. [...] Le biographe trie de quoi composer une forme qui ne ressemble à aucune autre. Il n'est pas utile qu'elle soit pareille à celle qui fut créée jadis par un dieu supérieur, pourvu qu'elle soit unique, comme toute autre création ${ }^{52}$.

des récits brefs qui concentrent leur action autour d'une crise et qui font surgir, grâce à quelques traits rapidement esquissés, des personnages à la fois uniques et énigmatiques, l'auteur rappelle que la fiction est d'abord la représentation dynamique et densifiée du réel. Grâce à son exclusivité et à son pouvoir suggestif qui la soustrait à son simple contenu empirique, l'image contribue à rappeler la "singularité quelconque » de tout être humain. Comme le fait remarquer Alexandre Gefen, Schwob élabore une nouvelle définition de l'art biographique que nous pourrions ainsi étendre, nous semble-t-il, à sa conception du réalisme en général :

C'est un coup de force majeur, à la fois théorique et pratique, pour l'histoire et la logique des genres littéraires : renversant l'opposition aristotélicienne qui attribue le particulier à l'histoire et le domaine des vérités générales à la littérature, refusant de faire du particulier le domaine de l'ineffable, Schwob reverse le genre de la vie à la littérature et légitime l'intrusion de l'imaginaire dans la biographie ${ }^{53}$.

La forme du récit bref, par sa poétique du fragmentaire et de la paralipse ${ }^{54}$, contribue évidemment au phénomène de l'omission : de ce roi au masque d'or, sans nom propre et sans histoire, nous ne savons rien. Seul un attribut - si ambigu soit-il - tel qu'un masque d'or s'étant mué en masque de lèpre permet de le singulariser parmi ses sujets. Que savons-nous de Matteo, ce "compagnon », "grand joueur de dés ${ }^{55}$ ", avec qui le 
narrateur de «La Peste» fuit l'épidémie florentine et qui trouvera une fin tragique dans les geôles papales, victime des symptômes de la peste ? ou de ce frère, porteur des marques du choléra, que le narrateur du « Train 081 » a perdu de vue depuis son départ en Chine :

Mon frère [...] avait pris la flotte. Il était dans les machines des transports. Il était entré là-dedans avant 1860, la campagne de Chine. Et la guerre finie, je ne sais comment il était resté dans le pays jaune, vers une ville qu'on nomme Canton. Les Yeux-Tirés l'avaient gardé pour leur mener des machines à vapeur. Sur une lettre que j'avais reçue de lui en 1862, il me disait qu'il était marié, et qu'il avait une petite fille $e^{56}$.

Inutile encore de préciser qu'un récit tel que «Les Sans-Gueule » porte l'énigme de ses personnages jusque dans son titre: deux êtres sans visage, et donc sans histoire. Le « déficit de savoir » engage ainsi le lecteur sur la voie de la conjecture.

Mais par effet de contraste, les non-dits qui entourent la description des personnages renforcent le pouvoir des images. Si celles-ci ont selon Schwob une «tendance hallucinatoire ${ }^{57}$ ", c'est bien parce qu'elles ne remplissent pas le rôle de simples illustrations, renvoyant à un référent quelconque. L'expression est en effet importante, puisque " halluciner » dérive du grec aluein qui signifie " errer ", " être agité, hors de soi » : « une image littéraire, c'est un sens à l'état naissant ${ }^{58}$ ", dit justement Bachelard. Si l'image traduit la dynamique de la vie, c'est parce qu'elle présente une valeur transfigurative ${ }^{59}$.

Dans son étude sur le fonctionnement de l'imaginaire, le philosophe Jean-Jacques Wunenburger rappelle la réalité pragmatique des images puisqu'elles « forment [...] des ensembles vivants qui se structurent, se transforment, interagissent, et par là sont à même de solliciter notre attention, d'aiguillonner nos affects, d'infléchir notre pensée ${ }^{60}$ ». L'image dépasse alors le stade empirique de simple signe du réel, grâce à sa " capacité d'élargissement des significations ${ }^{61}$ ». Celle-ci peut s'appuyer sur deux types de processus. Le premier, que Schwob décrit justement, relève de l'analogie: «[...] l'image d'une réalité perçue, qui est insérée dans un flux d'images de conscience, peut raviver des images associées, par ressemblance ou par contiguïtét ${ }^{62}$." N'est pas seulement concédé au lecteur, néanmoins, le droit de procéder à une lecture sensible, émotionnelle et subjective de l'œuvre ; Schwob conçoit que le texte impose aussi en luimême des représentations qui, obligeant ce même lecteur à reconsidérer sa vision du réel, active un processus herméneutique; ceci correspond au second processus sur lequel s'appuie l'image et qui « active, sur un plan paradigmatique, une chaîne d'images qui se relient les unes aux autres par un sens commun ${ }^{63} »$. Fonctionnant comme un symbole, "l'image ne renvoie plus seulement au signifié dominant, qui définit sa signification (Bedeutung) littérale, mais à un signifié indirect, caché, auquel on accède par une orientation de sens $(\operatorname{Sinn})^{64} »$. L'image symbolique « se tient au carrefour d'une intentionnalité herméneutique, apte à passer au-delà du sensible, et d'une configuration intrinsèque de l'image qui porte l'empreinte d'un sens encore en amont ${ }^{65}$ ».

29 En choisissant justement de composer ses récits à partir d'images « irréelles ", c'est-àdire d'images dont l'étrangeté et l'ambiguïté activent la participation du lecteur, Schwob donne une haute importance au « code » herméneutique, selon la terminologie établie par Barthes dans $S / Z$. Ce qui invite en effet à rapprocher nombre de récits schwobiens de la catégorie du fantastique - au-delà des thématiques évidentes qu'ils peuvent aborder et de l'angoisse qu'ils font parfois naître chez le lecteur-, ce sont 
essentiellement l'ambiguïté et l'impossible clôture du sens sur lesquelles ils reposent tous $^{66}$. Développant ses intrigues autour de personnages au profil incertain et dont les blessures ou cicatrices se lisent comme des marqueurs de différenciation tout autant que d'indifférenciation, Schwob n'offre au lecteur que les indices du caractère fragile et relatif du sens. Chaque nouvelle interprétation qui vient se superposer à une autre invite moins à se méfier du sens qu'à le supposer en perpétuel devenir. Engageant la liberté du signe, Schwob place ainsi au cœur de son projet littéraire le questionnement philosophique :

Tout poème, - et je prends le mot dans son acception la plus étendue, - qui contient une parcelle de vraie vie a pour refrain ce : «Marche ! marche! » comme si c'était sa qualification nécessairement sous-entendue et sans laquelle il perd tout sens réel. [...] Oui, le marcheur éternel erre dans ces pages de son pas inquiet. Et le livre aujourd'hui qui touche nos cœurs ne commence en vérité que là où son récit finit, - et pour être continué par nous, en nous ${ }^{67}$.

\section{L'image et la « sensation du détail unique »}

S'il est un terme qui permet de tirer un fil rouge entre les trois grandes préfaces composées par Schwob, c'est peut-être celui de mimèsis, compris dans le sens de représentation littéraire de la réalité ${ }^{68}$. Rappelons qu'il est question dans ses écrits théoriques et dans son œuvre fictionnelle de donner à voir et d'interroger un réel en crise. Schwob se démarque largement de la conception aristotélicienne de la représentation qui voit en la littérature le medium par lequel sont exposées les vérités générales du monde. L'auteur participe ainsi à un mouvement de critique, trouvant son acmé chez les avant-gardes du $\mathrm{xx}^{\mathrm{e}}$ siècle, à l'égard d'une prétention purement imitative de la représentation. L'heure n'est pas encore venue de nier à la littérature toute vertu figurative et de tendre à un art qui s'interdirait toute entreprise descriptive : caractère de l'œuvre dite présentative qui «ne renvoie qu'à elle-même, par opposition à l'œuvre représentative ${ }^{69} »$. Et pourtant, il ne fait pas de doute, comme le reconnaît l'ensemble de la critique schwobienne, que par sa volonté affichée d'émousser la confiance en la toute-puissance de l'intelligible et de tourner son regard vers la seule présence et contingence du réel, cette poétique annonce le siècle qui suit, surtout dans sa seconde moitié.

31 Si par « impressionnisme », l'artiste entend « imiter la nature dans les formes que nous saisissons en elle ", la représentation est donc d'abord le reflet d'une vision singulière, d'un imaginaire propre à l'artiste dans lequel le lecteur se trouve emporté. À propos du recueil des Hespérides du poète anglais Robert Herrick, Schwob décrit l'émotion synesthésique que la lecture poétique permet d'éprouver :

Lire Herrick, c'est lire des abeilles et du lait. Les mots sont luisants d'huile de fleurs,

frottés de nard et diaprés de gouttelettes parfumées. Ses vers volent à l'éternité avec de petites ailes d'or battu. Il ne faut pas plus qu'ouvrir les Hespérides et y tremper vite les yeux comme dans une vapeur de benjoin. Toute ligne apparue est peinte d'odeur qu'on hume du regard. [...] Mais ne lisez pas longtemps : vous seriez noyé dans un océan de roses ${ }^{70}$.

Wolfgang Iser considère qu' ' une description de l'interaction entre le texte et le lecteur doit se rapporter d'abord au processus de constitution par lequel le texte est vécu en étant lu», "une telle expérience [étant] nécessairement antérieure à l'attribution d'une signification à l'œuvre, puisqu'elle lui sert de fondement ${ }^{71} »$. Chez un écrivain qui 
insiste autant sur les impressions que procure la lecture et sur le potentiel imaginatif du texte, cette remarque fait sens d'une manière toute particulière.

En Stevenson, l'un des auteurs qu'il admire avec le plus de ferveur ${ }^{72}$, Schwob découvre un artiste qui «n'a jamais regardé les choses qu'avec les yeux de son imagination ${ }^{73}$ ", « his mind's $e y e^{74} »$. L'œil imaginatif de l'écrivain permet ainsi de multiplier les perspectives et d'entrevoir les visages les plus différenciés. Afin de donner à pressentir de l'unicité de l'individu dans le flux de la masse, l'artiste doit trier dans la matière du vivant, "créer", comme nous l'avons vu, "dans un chaos de traits humains». Si Schwob ne peut concevoir la représentation de la vie humaine sans qu'il soit fait mention de son «milieu », ce n'est pas parce que l'espace détermine le caractère d'un individu, mais parce que, comme le soulignait déjà Remy de Gourmont, le contre-point qu'offre l'arrière-plan permet d'en faire émerger la singularité :

Le génie particulier de M.Schwob est une sorte de simplicité effroyablement complexe; c'est-à-dire, que par l'arrangement et l'harmonie d'une infinité de détails justes et précis, ses contes offrent la sensation d'un détail unique ; il y a dans la corbeille de fleurs une pivoine que seule on voit parmi les autres abolies, mais si les autres fleurs n'étaient pas groupées autour d'elle, on ne verrait pas la pivoine ${ }^{75}$.

On ne saurait peut-être trouver meilleur résumé de l'esthétique schwobienne, car l'art symétrique est aussi un art du contre-point. Ce qui fait forte impression chez le lecteur et lui donne «la sensation du détail unique ", c'est un maniement tout à fait judicieux de l'image du trait corporel, coup de pinceau de l'artiste susceptible de faire pressentir l'unicité des référents humains.

35 À la croisée du réel et de l'irréel, l'image s'appuie selon Schwob sur les données concrètes de la perception tout en obéissant à des lois qui lui sont propres. C'est de cette particularité que jouit l'image littéraire dont l'objectif «n'est pas tant de faire violence au réel, que de le parachever ou de le "sublimer" dans un surréel qui en exprime la pulpe, la forme secrète ${ }^{76} »$; Schwob reconnaît d'ailleurs chez les plus grands écrivains le génie du maniement de l'image et de son pouvoir de suggestion : dans son essai principal consacré aux romans de Stevenson, il tente de saisir ce qui est à la source de l'originalité imaginative de l'écrivain écossais. Ce faisant, il nous renseigne vraisemblablement - Christian Berg ${ }^{77}$ le notait déjà - sur ses propres intentions esthétiques.

Conviant les sens, les images autour desquelles Stevenson « coule son histoire ${ }^{78}$ » sont source pour le lecteur d'une « espèce d'émoi d'imagination ${ }^{79}$ " que Schwob expérimente pour la première fois à la lecture de Treasure Island : «Alors je connus que j'avais subi le pouvoir d'un nouveau créateur de littérature et que mon esprit serait hanté désormais par des images de couleur inconnue et des sons point encore entendus ${ }^{80}$. $"$ Ce qui caractérise ainsi l'imaginaire de Stevenson, c'est le «jamais vu ni lu ${ }^{81} »$. En effet, ces images frappent le regard, précisément parce que leur objet est singularisé avec une intensité qu'il n'est guère possible de capter dans un réel soumis à des lois d'une autre nature :

Nous avions trouvé chez bien des écrivains le pouvoir de hausser la réalité par la couleur des mots; je ne sais pas si on trouverait ailleurs des images qui, sans l'aide des mots, sont plus violentes que les images réelles. Ce sont des images romantiques, puisqu'elles sont destinées à accroître l'éclat de l'action par le décor ; ce sont des images irréelles, puisqu'aucun œil humain ne saurait les voir dans le monde que nous connaissons. Et cependant elles sont, à proprement parler, la quintessence de la réalité ${ }^{22}$. 
Iréalité correspond pour Schwob à la « quintessence de la réalité ", c'est parce que loin d'être une "erreur », elle contient une énergie vitale qui dépasse celle que l'on peut percevoir dans le monde matériel. De surcroît, l'écrivain considère que la vie humaine ne peut être exprimée qu'à la condition d'en faire ressentir le caractère individuel. Il remarque ainsi qu'en choisissant délibérément de peindre des images où le factice se manifeste sans détour, Stevenson construit des personnages qui frappent l'esprit avec une intensité toute particulière :

En effet, ce qui reste en nous d'Alan Breck, de Keawe, de Thevenin Pensete, de John Silver, c'est ce pourpoint aux boutons d'argent, cette tache irrégulière de lichen, stigmate de la lèpre, ce crâne chauve avec sa double touffe de cheveux rouges, cette face large comme un jambon, avec les yeux scintillants comme des éclats de verre. N'est-ce pas là ce qui le dénote dans notre mémoire ? ce qui leur donne cette vie factice qu'ont les êtres littéraires, cette vie qui dépasse tellement en énergie la vie que nous percevons avec nos yeux corporels $[\ldots]^{83}$.

Romantiques et irréelles, les images prennent donc une teinte bachelardienne : elles sont littéraires parce qu'elles aspirent à la sublimation de la réalité. D'où l'aura poétique qui entoure aussi bien les romans de Stevenson que les contes de Schwob; les images que ces textes véhiculent appellent à une quête du sens et transportent le lecteur dans un univers autonome qui, pour être le fruit du langage, donne pourtant un accès privilégié au réel. En rencontrant l'imaginaire de Stevenson, Schwob fait la découverte d'un principe épiphanique qui régit le langage poétique et lui confère la liberté d'exprimer des relations et des correspondances qui sortent du cadre de la stricte logique ; par le pouvoir sensible et suggestif du verbe, le texte poétique devient le témoin du caractère singulier du monde :

Il me semble que les personnages de Stevenson ont [...] cette espèce de réalisme irréel. La large figure luisante de Long John [personnage de Treasure Island (1883)], la couleur blême du crâne de Thevenin Pensete [personnage de A Lodging for the Night: A Story of Francis Villon (1877)] s'attachent à la mémoire en vertu de leur irréalité même. Ce sont des fantômes de vérité, hallucinants comme de vrais fantômes. Notez en passant que les traits de John Silver hallucinent Jim Hawkins, et que François Villon est hanté par l'aspect de Thevenin Pensete ${ }^{84}$.

En excédant la capacité de perception propre au monde physique, l'objet imaginaire prend ainsi selon Wolfgang Iser un caractère inédit :

[L'imagination visuelle n'est pas] une vision optique au sens propre du mot, mais bien [une] tentative de se représenter ce que l'on ne peut jamais voir en tant que tel. Le caractère particulier de ces images consiste en ceci, qu'elles font apparaître des aspects qui n'auraient pu s'imposer à la perception directe. L'imagination visuelle présuppose l'absence matérielle de ce qui apparaît dans l'image ${ }^{85}$.

Puisqu'il est question d'animer des images et de les doter d'une vie dont l'énergie transcende le réel, il est dès lors peu surprenant que les impressions les plus fortes naissent chez Stevenson - et chez Schwob également - de la représentation des individus : " nous ne pouvons ", selon Schwob, " modeler personne à leur image, parce qu'elle est trop vive et trop singulière, ou qu'elle est liée au costume, à un jeu de lumière, à un accessoire de théâtre, pourrait-on dire ${ }^{86} »$. Pour illustrer ce propos, l'écrivain s'appuie sur une anecdote étonnante : évoquant sa participation à la mise en scène de 'Tis pity she's a whore de John Ford, il se rappelle que pour figurer le cœur sanglant d'Annabella planté sur le poignard de Giovanni, on s'était servi aux répétitions d'un véritable organe de mouton. Or, rien ne semblait plus artificiel et plus grossier que cet objet pourtant parfaitement réaliste. On avait pensé alors «que, puisqu'un vrai 
cœur paraissait faux en scène, un faux cœur devait paraitre vrai ${ }^{87}$ » et la troupe avait décidé de remplacer le cœur de mouton par un morceau de flanelle rouge vif :

Quand nous vîmes paraître une seconde fois Giovanni avec sa dague, nous eûmes tous un petit frémissement d'angoisse, car c'était bien là, à n'en pas douter, le cœur sanglant de la belle Annabella ${ }^{88}$.

41 Irréelle - non parce que factice, mais parce que produit d'une création - l'image que renvoient les personnages de Stevenson se trouve rehaussée d'une lumière particulière : ceux-ci sont «stigmatisés si vivement par son réalisme romantique que nous risquons fort de ne jamais les rencontrer ici-bas ${ }^{89}$ ». En baignant ses figures dans une nébuleuse mystérieuse, l'écrivain écossais focalise ainsi le regard sur un nombre limité d'éléments qui renforcent en même temps le caractère étranger et singulier de la représentation; une seule image, quelques traits permettent de marquer les individus du sceau de la singularité. De manière générale, nous remarquons ainsi que l'écrivain tend à animer et à singulariser ses personnages en les particularisant à même la surface de leur corps. Les portraits que l'on rencontre dans l'œuvre se résument le plus souvent à l'évocation de quelques traits particuliers, "brossés », comme chez Stevenson, «en trois touches de pinceau » et qui évoquent des altérations physiques majeures ou des empreintes, qu'elles soient des marques, des taches, des cicatrices ou encore des blessures. Les détails ne sont pas utilisés, comme nous allons le voir, dans le dessein de figurer la décomposition et la fragmentation du corps, mais, au contraire, dans celui de ramener l'image à l'unicité première de l'objet qu'elle représente.

Dotée d'une vertu hallucinatoire, l'image fonctionne donc à proprement parler sur le mode du surgissement, ce qui lui confère les propriétés de l'hypotypose. Plus précisément, en introduisant la caractérisation au sein du discours, elle offre "une série de détails, une vision fragmentaire composée d'une succession de sensations précises $^{90} »$. Chez Stevenson comme chez Schwob pourtant, ce procédé ne renvoie pas à une vision incomplète des personnages, mais ramène bien plutôt le référent à son unicité ; l'image-hypotypose est utilisée afin d'opérer une synthèse ; "embrasser tous les détails d'un seul regard", voilà à quoi pourrait aspirer l'image schwobienne que nous décrit Bijvanck :

L'action y est resserrée en un seul moment et l'exposition même renferme la crise. La clarté de l'ensemble, ni la précision des détails n'en souffrent pourtant. L'auteur semble nouer le nœud du récit, tandis qu'en réalité il en prépare la solution. Tout est fini en un clin d'œil. On se croirait en présence d'un escamoteur et c'est bien vraiment d'un artiste qu'il s'agit, car il a observé le moment précis où la crise vient de naître dans l'âme de ses personnages, et où, en se manifestant, elle appelle l'aventure qui amènera la catastrophe. À cet instant exactement il éclairera vivement le groupe de figures qu'il a choisi, et, le faisant ressortir en haut relief, il nous permet d'en embrasser tous les détails d'un seul regard. Il a su entasser ainsi dans l'espace le plus resserré une multitude de traits tout débordants de vie palpitante ${ }^{91}$.

43 Jean-Pierre Bertrand reconnaît d'ailleurs à cette écriture, pourtant composite et polyphonique, un trait récurrent, la rappelant de fait à des exigences poétiques :

D'une économie extrême, elle va droit à l'effet et sait rendre la présence troublante de tout un monde à partir d'un détail. Et c'est là l'un des grands mérites de cet auteur inclassable, que d'avoir fait passer le récit bref, du genre à la mode qu'il était, à une forme d'art pur souvent bien proche du poème en prose ; une forme qui dissout en elle toute prétention au roman ou à la poésie versifiée - genres que, de manière significative, Schwob n'aura jamais pratiqués ${ }^{92}$. 
44

ons ici un exemple en proposant une brève étude de "La Peste ", l'un des nombreux textes de sensibilité médiévale issus du corpus schwobien. Le narrateur de ce conte énigmatique, Bonacorso de Neri de Pitti, « gonfalonier de justice à la commune de Florence ", évoque les souvenirs de la grande peste qui se serait abattue sur la cité en 1374. Il décrit ainsi - avec un réalisme brutal qu'on reconnaît volontiers à Schwob, "sorte de voix blanche, aux antipodes de tout pathos ${ }^{93}$ " selon l'expression de Pierre Jourde - la violence de l'épidémie qui fait rage dans la ville de Florence :

[...] la peste dévastait la cité. La maladie était soudaine, et attaquait dans la rue. Les yeux devenaient brûlants et rouges, la gorge rauque; le ventre enflait. Puis la bouche et la langue se couvraient de petites poches pleines d'eau irritante. On était possédé par la soif. Une toux sèche secouait les malades pendant plusieurs heures. Ensuite, les membres se raidissaient aux articulations; la peau se parsemait de taches rouges, gonflées, qu'aucuns nomment bubons. Et finalement, les morts avaient la figure distendue et blanchâtre, avec des meurtrissures saignantes et la bouche ouverte comme un cornet ${ }^{94}$.

Dans cette description terrible et sans complaisance, le regard porte sur des corps pestiférés, soumis à un mal dont la progression implacable est explicitement exprimée par des indices temporels (« soudaine», «puis», "pendant», « ensuite », « et finalement »). Yeux, gorge, ventre, bouche, langue, articulations, peau, figure : chaque membre, chaque fragment du corps devient le support sur lequel se dessinent les indices d'une maladie dotée d'une existence autonome («attaquait dans la rue »). La souffrance qu'elle engendre traverse la chair et modifie, avec une rapidité fulgurante et jusqu'à l'anéantir, l'apparence corporelle et les traits du visage (« les morts avaient la figure distendue et blanchâtre »). La brutalité de la syntaxe, des tropes et du lexique surtout, qui inspire tout à la fois le dégoût et la pitié à l'égard de ces êtres réduits à la fonctionnalité de leurs attributs corporels, renforce le caractère aliénant de la peste : celle-ci se manifeste ainsi par des gonflements, des raidissements, des brûlures et des irritations qui rythment le spectacle macabre de l'agonie. Puis la description prend du recul et donne à voir le chaos qui règne dans les rues de la cité toscane :

Les fontaines publiques, presque épuisées par la chaleur, étaient entourées d'hommes courbés et maigres qui tâchaient d'y plonger la tête. Plusieurs s'y précipitèrent et on les retirait par les crochets des chaînes, noirs de vases et le crâne fracassé. Les cadavres brunissants jonchaient le milieu des voies par où coule, dans la saison, le torrent des pluies : l'odeur ne pouvait se supporter et la crainte était terrible ${ }^{95}$.

L'élargissement du champ de vision renforce encore le caractère indifférencié des pestiférés dont on ne distingue que la silhouette funeste : ils ne sont déjà plus que des " hommes courbés et maigres" avant que la mort ne les rattrape et ne les réduise à l'état de « cadavres brunissants ».

C'est cette vision lugubre qui inspire la fuite au narrateur et à son compagnon Matteo. Ensemble, ils traversent une campagne hantée par la peur de l'épidémie. Jetés injustement dans un cachot par les soldats du Pape avignonnais et menacés par le bûcher, ils tentent de mettre au point un subterfuge pour s'évader. L'idée leur vient " que la justice papale, par terreur de la maladie, [les] fer[a] jeter dehors ${ }^{96}$ ». Aussi est-il décidé de recouvrir le visage de Matteo de polenta et de simuler les râles du pestiféré pendant que Bonacorso appellera à l'aide. Mais qu'advient-il lorsque le masque, artifice et attirail de folklore, se fait chair?

[...] cependant, Matteo continuait à geindre. Je le poussai du coude, afin qu'il se reposât jusqu'au jour: ses gémissements devinrent plus forts. Je le touchai dans 
l'obscurité : mes mains n'atteignaient que son ventre qui me parut gonflé comme une outre. coulevée par de hideux gonflements ${ }^{100}$ " qu'entrevoit le roi au masque d'or dans le miroir de la rivière, ou à la " petite bande blanchâtre irrégulière, de couleur crayeuse ${ }^{101}$ ", signe d'une lèpre que croit reconnaître le narrateur de "L'Origine ». Et alors que sur le visage de Matteo, Bonacorso identifie «les croûtes blanches et le suintement rouge de la peste de Florence ${ }^{102}$ », le narrateur du «Train 081 » aperçoit sur le corps de son frère les marques du choléra, «plaques bleuâtres tach[ant] sa peau » et « ses yeux [...] entourés de cercles bleus ${ }^{103}$ ». Quant à la sœur Angèle de "L'Hôpital », elle reconnaît Julot l'Oreille-Mangée à "son moignon d'oreille », « une dentelure de chair cicatrisée, souvenir d'un "dos [souteneur] qui lui avait bouffé l'esgourde [l'oreille]" ${ }^{104} »$. On se gardera du reste de faire encore le décompte des altérations qui recouvrent la « double cicatrice arrondie, gigantesque et sans signification » des Sans-Gueule. Il serait facile d'invoquer bien d'autres occurrences témoignant de la rigueur - confinant à l'obsession - avec laquelle Schwob travaille ce motif.

51 Dans plusieurs de ses contes, Schwob appose ainsi sur les êtres des meurtrissures, signes de l'interaction permanente entre les individus : durablement, la surface garde le souvenir du passage d'autrui. Nous savons, grâce à Jacques Fontanille, que l'empreinte, opérant comme mémoire figurative, est ainsi tout à la fois un «signifiant des interactions révolues ${ }^{105}$ » et « un signifiant en quête de son signifié ${ }^{106}$ ». En tant que tel, le réseau des signes qui se dessine à la surface du corps en est la signature individuelle. Quoique déstabilisée, l'individualité du personnage s'en trouve néanmoins renforcée dans la mesure où ces empreintes, transformant effectivement l'enveloppe 
en surface d'inscription, «stigmatis[ent]» littéralement les êtres: «de geste destructeur, l'altération de la surface peut cependant se transformer en geste refondateur ${ }^{107}$ ", indique à cet égard Maryline Maigron. Aussi la surface corporelle devient-elle chez Schwob le lieu où se manifeste la singularité de chaque être: les cicatrices qui recouvrent l'enveloppe sont les marques individuelles du sujet, matérialisant une mémoire qui garde le souvenir de ses interactions avec le monde extérieur et articulant des signes muets ${ }^{108}$ à interpréter. La trace ne pouvait ainsi mieux illustrer la tridimensionnalité du signe: mot, chose, et concept. Signe de signe, présence d'une absence, l'empreinte est le lieu d'une quête esthétique et éthique des réalités.

\section{NOTES}

1. M. Schwob, "Préface », Le Roi au masque d'or, dans CEuvres, S. Goudemare (éd.), Paris, Phébus, coll. «Libretto », 2002, p. 242. Désormais les notes renvoient à cette édition, désignée par CEuvres (sauf en cas de renvoi précisé à l'édition des CEuvres aux Belles-Lettres par A. Gefen).

2. A. Gefen, "Philosophies de Marcel Schwob ", dans Chr. Berg, A. Gefen, M. Jutrin et A. Lhermitte (dir.), Retours à Marcel Schwob: D'un siècle à l'autre (1905-2005), Presses universitaires de Rennes, 2007, p. 23. Dans la préface du Roi au masque d'or, il est dit que si « Dieu parle : alors l'univers est son langage » (M. Schwob, « Préface », Le Roi au masque d'or, dans EFuvres, éd. citée, p. 242).

3. Référence au poème «Je chante le soi-même » du poète américain Walt Whitman (1819-1892), texte liminaire du recueil Feuilles d'herbe (Leaves of Grass, 1855-1892), dont Schwob a traduit des extraits à vingt ans, à une époque où le poète américain est encore quasiment inconnu en France (la première traduction complète des Feuilles d'herbe date de 1909).

4. M. Schwob, « Préface ", Cour double, dans CEuvres, éd. citée, p. 50.

5. M. Schwob, « Préface », Vies imaginaires, dans Euvres, éd. citée, p. 511.

6. M. Schwob, « Préface », Cour double, dans CEuvres, éd. citée, p. 47.

7. G. Agamben, La communauté qui vient: théorie de la singularité quelconque, Paris, Seuil, coll. « La Librairie du XXI ${ }^{\mathrm{e}}$ siècle ", 1990, p. 10.

8. Dans la préface des Vies imaginaires, Schwob développe encore davantage cette idée. Il ne s'agira plus seulement de « décri[re] les aventures singulières [nous soulignons] de l'esprit et du corps ", mais de forcer le trait du singulier et "décrir[e] un homme en toutes ses anomalies " (M. Schowb, « Préface », Vies imaginaires, éd. citée, p. 510).

9. M. Schwob, « Préface », Cour double, dans CEuvres, éd. citée, p. 52.

10. J.-K. Huysmans, À rebours, Paris, Garnier-Flammarion, coll. « GF », 1978, p. 45.

11. M. Schwob, « Préface », Cour double, dans CEuvres, éd. citée, p. 50.

12. Ibid., p. 48.

13. Ibid., p. 45.

14. M. Schwob, «Le Réalisme » [1889], dans CEuvres, A. Gefen (éd.), Paris, Les Belles Lettres, 2002, p. 830.

15. Ibid.

16. M. Schwob, « Préface », Cour double, dans Euvres, éd. citée, p. 43.

17. Ibid.

18. Ibid., p. 46. 
19. Dérivé du verbe idein, qui sert, selon le dictionnaire d'Alain Rey, d'aoriste à horan « voir » et se rattache à une racine indo-européenne *weid- "voir, percevoir par la vue », que l'on retrouve dans le latin video et certaines formes germaniques, comme l'anglais witnes (A. Rey, Dictionnaire historique de la langue française [1992], Paris, Dictionnaire Le Robert-Sejer, 1998, p. 1773).

20. M. Schwob, « Préface ", Cœur double, dans CEuvres, éd. citée, p. 45.

21. M. Schwob cité dans W. G. C. Bijvanck, Un Hollandais à Paris en 1891 : sensations de littérature et d'art, Paris, Perrin, 1892, p. 191.

22. Chr. Berg, "Marcel Schwob, le récit bref et l'esprit de symétrie ", dans K. Gyssels, S. Hillen, L. Rasson et I. Van Acker (éds), Christian Berg, L'Automne des idées. Symbolisme et décadence à la fin du XIX siècle en France et en Belgique, Louvain, Peeters, coll. « La République des Lettres », 2013, p. 223.

23. M. Schwob, « Robert Louis Stevenson » [1894], Spicilège et autres essais, dans Cuvres, éd. citée, p. 728.

24. M. Schwob, «Dix leçons sur François Villon», dans Euvres complètes, P. Champion (éd.), Genève-Paris, Slatkine Reprints, 1985, p. 295-296.

25. Ibid., p. 44.

26. Outre ses activités d'écrivain et de journaliste, Schwob s'est également illustré par ses traductions d'auteurs tels que Shakespeare, Stevenson, De Foe, Meredith, etc.

27. M. Schwob, préface à " "La tragique histoire d'Hamlet" de William Shakespeare", trad. E. Morand et M. Schwob, Spicilège et autres essais, dans CEuvres, éd. citée, p. 837.

28. M. Schwob, « Paul Verlaine », dans M. Schwob, Euvres, A. Gefen (éd.), Paris, Les Belles Lettres, 2002, p. 870.

29. «Du réel au possible, il y a la distance d'un nom », dit Remy de Gourmont à propos de l'œuvre de Schwob (Le deuxième livre des masques, Paris, Mercure de France, 1923, p. 153).

30. M. Schwob, «Le Réalisme » [1889], dans CEuvres, A. Gefen (éd.), Paris, Les Belles Lettres, 2002, p. 830.

31. M. Schwob, Cour double. Le Livre de Monelle, J.-P. Bertrand (éd.), Paris, Flammarion, coll. « GF », 2008, « Présentation », p. XVI.

32. Y. Vadé, Le poème en prose et ses territoires, Paris, Belin, coll. « Lettres Belin Sup », 1996, p. 207.

33. B. Vibert, Poète, même en prose, Paris, Presses universitaires de Vincennes, coll. «L'Imaginaire du Texte », 2010, p. 40.

34. M. Schwob, « Préface », Le Roi au masque d'or, dans CEuvres, éd. citée, p. 243.

35. C. Rosset, L'objet singulier [1979], Paris, Les Éditions de Minuit, coll. «Critique », 1985, p. 16.

36. Chr. Berg, "Signes de signes, Marcel Schwob et le "rapport mystérieux des signes" ", dans Chr. Berg et Y. Vadé (dir.), Marcel Schwob d'hier et d'aujourd'hui, Seyssel, Champ Vallon, coll. « Détours ", 2002, p. 104.

37. Chr. Berg insiste sur le fait que les signes «n'existent que grâce au système de renvoi et de signification dans lequel ils sont censés fonctionner » (ibid., p. 241).

38. M. Schwob, « "Le Démon de l'Absurde” de Rachilde, préface ", dans CEuvres, éd. citée, p. 811.

39. M. Schwob, « Robert Louis Stevenson » [1894], Spicilège et autres essais, dans CEuvres, éd. citée, p. 725-726.

40. "Tout l'effort de Schwob a été de se libérer du réalisme au nom d'un réalisme supérieur. »

(G. Trembley, Marcel Schwob, faussaire de la nature, Genève, Droz, 1969, p. 87.)

41. M. Riffaterre, "L'illusion référentielle ", dans G. Genette et T. Todorov (dir.), Littérature et réalité, Paris, Seuil, coll. « Points ", 1982, p. 92.

42. Chr. Berg observe que "Schwob [...] a recours à la vieille ficelle de l'“observateur venu d'un autre monde" afin de montrer la relativité des concepts en présence » («Signes de signes, Marcel Schwob et le "rapport mystérieux des signes" ", art. cité, p. 233). Pour une autre lecture de cette préface, voir B. Vibert, ouvr. cité, p. 127-129.

43. M. Schwob, « Préface », Le Roi au masque d'or, dans CEuvres, éd. citée, p. 240.

44. Ibid., p. 242. 
45. Grâce aux témoignages de certains de ses proches, il paraît presque assuré que le linguiste, polyglotte et féru de littérature nord-européenne qu'était Marcel Schwob, connaissait l'œuvre de Nietzsche, et cela bien avant que le travail du philosophe ne soit traduit en France. Dans « Marcel Schwob parmi ses livres ", Pierre Champion indique que l'écrivain Paul Gsell « se souvient bien de l'avoir entendu [Marcel Schwob] traduire Nietsche [sic], alors inconnu en France, sur le texte allemand » et que « cela était d'une rare beauté » (P. Champion [éd.], Catalogue de la bibliothèque de Marcel Schwob, Paris, Allia, 1993, p. 20). John Alden Green indique quant à lui - selon M. Delay dans La jeunesse d'André Gide - qu'" en 1893, Schwob et Robert Henri traduisent Nietzsche pour le Mercure de France » (M. Schwob, Correspondance inédite, J. A. Green [éd.], Genève, Droz, 1985, p. 86).

46. M. Schwob, « Préface », Le Roi au masque d'or, dans CEuvres, éd. citée, p. 242.

47. Ibid., p. 242-243.

48. M. Schwob, préface à " "La tragique histoire d'Hamlet" de William Shakespeare", trad.

E. Morand et M. Schwob, Spicilège et autres essais, dans Euvres, éd. citée, p. 837.

49. M. Schwob, "Il Libro della mia Memoria ", Spicilège et autres essais, dans CEuvres, éd. citée, p. 964.

50. M. Schwob, « Robert Louis Stevenson » [1894], Spicilège et autres essais, dans Cuvres, éd. citée, p. 726.

51. Ibid., p. 964-966.

52. M. Schwob, « Préface », Vies imaginaires, dans Euvres, éd. citée, p. 514-515.

53. A. Gefen, « Paolo Uccello, peintre », Inventer une vie. La fabrique littéraire de l'individu, Bruxelles, Les Impressions Nouvelles, 2015, p. 85.

54. Définie par Gérard Genette comme la « rétention d'une information logiquement entraînée par le point de vue adopté » (Nouveau discours du récit, Paris, Seuil, coll. « Points Essais », 1983, p. 44).

55. M. Schwob, « La Peste », Le Roi au masque d'or, dans CEuvres, éd. citée, p. 272.

56. M. Schwob, « Le Train 081 », Cour double, dans CEuvres, éd. citée, p. 75-76.

57. M. Schwob, « La Perversité », Spicilège et autres essais, dans Euvres, éd. citée, p. 764.

58. G. Bachelard, «L'image littéraire », cité par L. Lavaud, L'image, Paris, Flammarion, coll. «GF/ Corpus », 1999, p. 182.

59. Ibid.

60. J.-J. Wünenberger, La vie des images, Presses universitaires de Grenoble, coll. « Bibliothèque de l'imaginaire », 2002, p. 7.

61. Ibid., p. 17.

62. Ibid., p. 18.

63. Ibid.

64. Ibid.

65. Ibid., p. 46.

66. Ce qui rapproche le fantastique schwobien du modèle théorique développé à propos d'Henry James par Christine Brooke-Rose (A Rhetoric of the Unreal. Studies in Narrative and structure, Cambridge, Cambridge University Press, 1981).

67. M. Schwob cité dans W. G. C. Bijvanck, ouvr. cité, p. 304-305.

68. Pour un tour d'horizon des diverses traductions et compréhensions de cette notion dans la pensée européenne, voir A. Gefen, La mimèsis, Paris, Flammarion, coll. « GF/Corpus », 2002.

69. Tzvetan Todorov renvoie à Étienne Souriau comme fondateur de cette distinction: «C'est Étienne Souriau qui, dans sa Correspondance des arts (1947; $2^{\mathrm{e}}$ édition corrigée, que je cite, de 1969) a posé de la façon la plus explicite le problème de la représentation en art, en en faisant un trait distinctif et typologique. C'est qu'en effet, à côté des arts représentatifs, il en existe d'autres qui ne le sont pas, et auxquels Souriau donne le nom de "présentatifs" " («La poésie sans le vers », dans T. Todorov, Les genres du discours, Paris, Seuil, 1978, p. 126). 
70. M. Schwob, «Il Libro della mia Memoria », Spicilège et autres essais, dans Cuvres, éd. citée, p. 964.

71. W. Iser, L'acte de lecture : théorie de l'effet esthétique, Bruxelles, Pierre Mardaga, 1985, p. 50.

72. Il lui a consacré trois articles, a préfacé Le Dynamiteur et est l'auteur de nombreuses traductions qui permirent de faire connaître Stevenson au public français.

73. M. Schwob, « Robert Louis Stevenson » [1894], Spicilège et autres essais, dans CEuvres, éd. citée, p. 727.

74. Ibid., p. 731.

75. R. de Gourmont, ouvr. cité, p. 157.

76. L. Lavaud, L'image, Paris, Flammarion, coll. «GF/Corpus », 1999, p. 21.

77. Chr. Berg, «Signes de signes, Marcel Schwob et le "rapport mystérieux des signes", art. cité, p. 243.

78. M. Schwob, « Robert Louis Stevenson » [1894], Spicilège et autres essais, dans Cuvres, éd. citée, p. 730.

79. Ibid., p. 723.

80. Ibid., p. 723-724.

81. M. Schwob, « Robert Louis Stevenson » [1890], dans Euvres, A. Gefen (éd.), Paris, Les Belles Lettres, 2002, p. 831.

82. M. Schwob, « Robert Louis Stevenson » [1894], Spicilège et autres essais, dans CEuvres, éd. citée, p. 728.

83. Ibid., p. 728.

84. Ibid., p. 730.

85. W. Iser, ouvr. cité, p. 248.

86. M. Schwob, « Robert Louis Stevenson » [1894], Spicilège et autres essais, dans CEuvres, éd. citée, p. 729.

87. Ibid., p. 730.

88. Ibid.

89. Ibid., p. 729.

90. D. Bergez, V. Géraud et J.-J. Robrieux, Vocabulaire de l'analyse littéraire [1994], $2^{\mathrm{e}}$ éd., Paris, Armand Colin, 2010, p. 144.

91. W. G. C. Bijvanck, ouvr. cité, p. 244-245.

92. J.-P. Bertrand, « Présentation », éd. citée, p. VIII.

93. P. Jourde, "L'amour du singulier », dans M. Schwob, Euvres, A. Gefen (éd.), Paris, Les Belles Lettres, 2002, p. 32.

94. M. Schwob, « La Peste », Le Roi au masque d'or, dans Euvres, éd. citée, p. 271.

95. Ibid., p. 271-272.

96. Ibid., p. 274.

97. Ibid., p. 275.

98. W. G. C. Bijvanck, ouvr. cité, p. 234.

99. P. Jourde, «L'amour du singulier », dans M. Schwob, CEuvres, A. Gefen (éd.), Paris, Les Belles Lettres, 2002, p. 35.

100. M. Schwob, «Le Roi au masque d'or ", Le Roi au masque d'or, dans Euvres, éd. citée, p. 249.

101. M. Schwob, «L'origine », Contes de L'Écho, dans CEuvres, éd. citée, p. 666.

102. M. Schwob, «La Peste », Le Roi au masque d'or, dans Æuvres, éd. citée, p. 275.

103. M. Schwob, « Train 081 », Couur double, dans Euvres, éd. citée, p. 78.

104. M. Schwob, « L'Hôpital », Cœur double, dans Euvres, éd. citée, p. 209.

105. J. Fontanille, Corps et sens, Paris, PUF, 2011, p. 104.

106. Ibid., p. 107.

107. M. Maigron et M.-O. Salati (dir.), La Surface : accidents et altérations, Chambéry, Université de Savoie, 2010, p. 14. 
108. Rappelons qu'une étymologie incertaine relie "mot» et «muet» à travers la racine commune mutus.

\section{RÉSUMÉS}

Comprendre, comme Lucrèce, la variété des choses, c'est voir en chacune une épiphanie de l'unique, en présenter la "singularité quelconque » (Giorgio Agamben). Dans le conte poétique en prose tel que le pratique Schwob, ce regard induit un positionnement instable entre mimesis et absence de représentation. La lecture heuristique, détour trompeur mais indispensable avant une lecture herméneutique, privilégie chez l'auteur de Cœur double et du Roi au masque d'or l'émotion, l'effet, la "sidération ». Mais en même temps, et là réside la force des textes génériquement ambigus qui composent ces deux recueils, la «tendance hallucinatoire » que Schwob attribue à l'image déclinée comme hypotypose permet de saisir vivement l'unicité d'un référent particulier. Cette singularité à la fois quelconque et irréductible, qui transfère sur le monde référentiel le caractère non catégorisable que Benedetto Croce voyait dans l'objet esthétique, confère au « conte » schwobien une poétique aux contours changeants, imprévisibles. L'« effet-recueil » ne remet pas totalement en question a posteriori la singularité de chacun de ces textes prépubliés, et les préfaces elles-mêmes sont des cartes perverses, au sens propre, qui indiquent des pistes de lecture en même temps que leurs déviations.

Understanding the diversity of things, as Lucretius did, means seeing in each and every one of them an epiphany of uniqueness: exhibiting their "whatever singularity" (G. Agamben). In Schwob's practice of the prose poetic tale, this vision results in an unstable position between mimesis and the absence of representation. A heuristic reading, a deceiving but necessary detour on the way to hermeneutic reading, enhances emotions and stunning effects in the tales of Cour double and Le Roi au masque d'or. Yet at the same time, the "hallucinatory potency" which Schwob attributes to images treated as hypotyposes enables one to grasp the uniqueness of a particular referent: there lies the strength of these generically ambiguous texts in both collections. "Whatever singularity" is therefore made singular again, and what is peculiar to the aesthetic object according to B. Croce, namely the impossibility to categorize it, is transferred upon the referential world. The "collection effect" does not entirely question a posteriori the singularity of each of these pre-published texts, and even the author's prefaces seem to offer properly speaking "perverse" road maps by indicating ways of reading these narratives as well as alternative ways.

\section{INDEX}

Mots-clés : Marcel Schwob, contes poétiques en prose, Cœur double, Le Roi au masque d'or, idéalisme, antipositivisme, réalisme, impressionnisme, mimesis, semiosis, singularité, double, masque, image, imaginaire, émotion, sidération, Michael Riffaterre, Michel Bréal, Ferdinand de Saussure, Benedetto Croce, effet-recueil 
AUTEURS

PAULINE BLOCH

Université de Fribourg (Suisse)

MICHEL VIEGNES

Université de Fribourg (Suisse) 